\title{
Aspectos demográficos e socioeconômicos de mães atendidas em um programa de triagem auditiva neonatal
}

\section{Demographic and socioeconomic aspects of mothers attended in a newborn hearing screening program}

\author{
Silvana Maria Sobral Griz ${ }^{1}$, Camila Padilha Barbosa ${ }^{2}$, Adriana Ribeiro de Almeida e Silva ${ }^{3}$, Mariana Azevedo \\ Ribeiro ${ }^{4}$, Denise Costa Menezes ${ }^{5}$
}

\begin{abstract}
RESUMO
Objetivo: Descrever alguns aspectos demográficos e socioeconômicos de mães de neonatos e lactentes atendidos no serviço de Triagem Auditiva Neonatal, do Hospital das Clínicas, da Universidade Federal Pernambuco (HC-UFPE), nascidos no ano de 2007. Métodos: A coleta de dados foi constituída por leitura e digitação das informações contidas no banco de dados referente ao programa de Triagem Auditiva Neonatal do HC- UFPE. Participaram da pesquisa 1.193 mães. Resultados: Entre as variáveis demográficas do estudo destaca-se que o maior percentual (47,9\%) das mães tinha entre 20 e 29 anos de idade e 69,2\% eram casadas. Dentre as variáveis socioeconômicas, $36,1 \%$ possuíam o $1^{\circ}$ grau incompleto, 55,7\% eram donas de casa, 38,6\% não possuíam renda pessoal, 60,6\% dispunham de renda familiar entre um a três salários mínimos e 92,5\% tiveram seus filhos nascidos em maternidades públicas. Conclusão: A população estudada encontra-se em condições consideradas desfavoráveis para a saúde e desenvolvimento global dos neonatos e lactentes, pois as gestantes com este perfil pertencem a uma categoria de risco. Esses resultados servem de alerta para o desenvolvimento de ações que visem à promoção da saúde na população investigada.
\end{abstract}

Descritores: Triagem neonatal; Lactente; Audição; Perda auditiva; Fatores socioeconômicos; Características da população; Indicador de risco

\section{INTRODUÇÃO}

A perda auditiva neonatal, mesmo de grau leve, pode alterar o desenvolvimento e a aquisição da linguagem oral. Com o objetivo de alcançar melhores prognósticos em casos de perdas auditivas neonatais, programas de triagem auditiva neonatal (TAN) vêm sendo desenvolvidos em maternidades no Brasil e em diversos países ${ }^{(1-4)}$.

$\mathrm{O}$ crescente avanço na área se deve às inúmeras publicações científicas que apontam para melhores condições de desenvolvimento infantil quando a intervenção é iniciada nos seis primeiros meses de $v_{i d a}{ }^{(1-5)}$.

Trabalho realizado no Hospital das Clínicas da Universidade Federal de Pernambuco - HC-UFPE - Recife (PE), Brasil, com auxílio PROPESQ PIBIC PROEXT.

(1) Doutora, Professora do Curso de Fonoaudiologia da Universidade Federal de Pernambuco - UFPE - Recife (PE), Brasil.

(2) Pós-graduanda (Mestrado) do Programa de Pós-graduação em Enfermagem da Universidade Federal de Pernambuco - UFPE - Recife (PE), Brasil.

(3) Fonoaudióloga clínica - Recife (PE), Brasil.

(4) Fonoaudióloga clínica - Recife (PE), Brasil.

(5) Doutora, Professora do Curso de Fonoaudiologia da Universidade Federal de Pernambuco - UFPE - Recife (PE), Brasil.

Endereço para correspondência: Silvana Maria Sobral Griz. Av. Flor de Santana, 189/301, Parnamirim, Recife (PE), Brasil, CEP: 52060-290. E-mail: sgriz@terra.com.br

Recebido em: 26/2/2009; Aceito em: 27/10/2009
Vários fatores devem ser levados em consideração para a implantação de um programa de triagem auditiva neonatal, entre eles, fatores demográficos e socioeconômicos de uma determinada população com potencial impacto na gravidez ${ }^{(3)}$, na saúde do feto/neonato e no desenvolvimento do sistema auditivo.

Entre os fatores demográficos que merecem ser discutidos, pode-se citar a faixa etária e o estado civil das mães. O crescente número de gestantes adolescentes nos dias de hoje ${ }^{(6)} \mathrm{vem}$ sendo apontado como um problema social em todo o mundo ${ }^{(7)}$. Quanto ao estado civil das púberes, estudos apontam que viver legalmente ou consensualmente em relação conjugal poderá influenciar no modo como a gestação será percebida e aceita ${ }^{(7-9)}$.

O nível de escolaridade, a ocupação, a renda pessoal e familiar, e os bens familiares são fatores socioeconômicos que refletem na saúde das mães ${ }^{(10,11)}$. Alguns estudos ${ }^{(11-13)}$ dispensam atenção especial ao nível de escolaridade da mãe, por considerar este aspecto como elemento básico, que reflete nos cuidados com a gestação e, consequentemente, para a saúde do recém-nascido.

Outros fatores são discutidos, tais como, a quantidade de filhos em uma família, a realização do pré-natal e o tipo do hospital de nascimento da criança ${ }^{(13-17)}$.

Segundo o Instituto Brasileiro de Geografia e Estatística $(\mathrm{IBGE})^{(18)}$, o número de filhos por mulher está diminuindo nos últimos anos. Porém, constata-se maior quantidade de 
filhos nas famílias menos favorecidas economicamente e redução entre as famílias com melhores condições de vida. $\mathrm{O}$ maior número de filhos está associado a implicações sociais como assistência pré-natal inadequada e maior chance de prática de aborto e risco de sequelas como as malformações congênitas ${ }^{(5,8,19,20)}$. O pré-natal é um procedimento destinado a orientar a promoção da saúde e bem-estar, propiciando também a realização de diagnósticos e tratamento de complicações que afetam as gestantes e seus filhos, durante e após a gestação ${ }^{(14)}$.

Ao se identificar o tipo do hospital (público ou privado) em que foi realizado o parto, pode-se fazer inferências sobre alguns aspectos dos serviços prestados ${ }^{(17)}$. No Brasil, o Sistema Único de Saúde (SUS) é um programa nacional, criado segundo princípios de Universalidade, Equidade e Integralidade, para atender, com excelência, qualquer cidadão brasileiro, sem diferenças sociais ou de qualquer ordem. No entanto, observa-se que há preferência dos usuários pelo atendimento privado $^{(21)}$. Isso porque parecem existir dificuldades de acesso ao Sistema Único de Saúde.

Com base no exposto, percebe-se a importância de estudos sobre aspectos socioeconômicos e demográficos de populações específicas, voltados para efetividade de programas de Triagem Auditiva Neonatal (TAN). Essas informações, escassas na literatura nacional, podem ser relacionadas à situação de saúde de cada região, e contribuem para a melhoria de um programa de TAN efetivo, pois representam uma importante base diagnóstica para as necessárias ações de gestão, assistência e ensino na área de saúde materno-infantil ${ }^{(11)}$.

O presente estudo teve como objetivo descrever aspectos demográficos e socioeconômicos de mães de neonatos e lactentes atendidos no serviço de Triagem Auditiva Neonatal, do Hospital das Clínicas, da Universidade Federal Pernambuco (HC-UFPE), nascidos no ano de 2007.

$\mathrm{O}$ conhecimento sobre aspectos relacionados à população atendida no HC-UFPE contribuirá para a viabilização de estratégias de promoção à saúde auditiva na população estudada, uma vez que a eficácia de tais programas depende da integração da família em todo o processo de diagnóstico da perda auditiva. A determinação de perfis desta natureza fornecerá subsídios para que programas de triagem auditiva neonatal sejam direcionados para as diferentes realidades, com o intuito de melhor sensibilização e acolhimento destas mães.

\section{MÉTODOS}

Esta pesquisa foi realizada a partir de dados obtidos no serviço de Triagem Auditiva Neonatal (TAN), da Maternidade do Hospital das Clínicas, da Universidade Federal de Pernambuco (HC-UFPE). Este é um Hospital vinculado ao SUS, sendo referência para gestantes de risco. No serviço de TAN realizam-se exames de segunda à quinta-feira, em neonatos e lactentes nascidos no referido Hospital ou em outros hospitais públicos e privados. Para todos os neonatos e lactentes atendidos, é realizado o preenchimento de um formulário de registro do paciente, dividido em duas sessões: (1) registro das informações referentes à situação demográfica e socioeconômica das famílias, e, (2) registro dos indicadores de risco presentes no pré-, peri- e pós-natal. Participaram desta pesquisa todas as mães de neonatos e lactentes, atendidos no referido serviço ( $\mathrm{N}=1193)$, nascidos no ano de 2007.

Foi realizado um estudo observacional, descritivo, transversal e com desenho do tipo seccional. Os dados referentes a esta população, contidos nos formulários, foram digitados e calculados no programa Statistical Package for the Social Sciences (SPSS), na versão 13.0. Para análise dos dados foram obtidas distribuições absolutas e percentuais. Foram analisadas as variáveis: (a) demográficas: (a1) idade materna e (a2) estado civil, e (b) socioeconômicas: (b1) escolaridade da mãe, (b2) ocupação da mãe, (b3) renda pessoal e familiar, (b4) número de filhos, (b5) acompanhamento pré-natal, e (b6) tipo de maternidade.

A pesquisa foi aprovada pelo Comitê de Ética em Pesquisa envolvendo seres humanos do Centro de Ciências da Saúde da Universidade Federal de Pernambuco (UFPE), sob número 0158.0.172.000-07. Todas as mães atendidas no serviço de TAN do HC-UFPE assinaram um Termo de Consentimento Livre e Esclarecido.

\section{RESULTADOS}

Na análise dos dados demográficos, observa-se que a idade materna variou entre 12 e 46 anos, com uma média de 24,89 anos e desvio padrão de 6,73 anos. Observa-se um maior percentual para mães com idade entre 20 e 29 anos (47,9\%, $\mathrm{N}=572)$, em comparação com percentuais de 24,6\% ( $\mathrm{N}=293)$ para mães com idades superiores e inferiores a esse intervalo. Um percentual de $2,9 \%(\mathrm{~N}=35)$ das mães entrevistadas não informou a idade. No que se refere ao estado civil, foi observado que $69,2 \%(\mathrm{~N}=825)$ das mães pesquisadas eram casadas ou possuíam união consensual, sendo $27,8 \%(\mathrm{~N}=332)$ solteiras. Um percentual de 3\% ( $\mathrm{N}=36$ ) não informou o estado civil.

Os resultados referentes às variáveis socioeconômicas encontram-se na Tabela 1. Destaca-se que 36,1\% ( $\mathrm{N}=431)$ das mães possuíam primeiro grau incompleto, 55,7\% ( $\mathrm{N}=664)$ relataram ser donas de casa, 38,6\% ( $\mathrm{N}=461)$ não possuíam renda pessoal, 60,6\% $(\mathrm{N}=723)$ referiu possuir entre um a três salários mínimos, para renda familiar, 51,6\% (N=616) das mães possuíam um filho, 92,7\% (N=1106) informou ter realizado acompanhamento pré-natal.

\section{DISCUSSÃO}

Buscou-se determinar a ocorrência de alguns aspectos socioeconômicos e demográficos, para que, em estudos futuros, esses dados possam ser relacionados à efetividade do programa de TAN da região. Número de falhas na TAN, retornos para o reteste e/ou número de indivíduos identificados com perda auditiva, segundo o $\mathrm{JCIH}^{(5)}$ são interessantes indicadores para avaliação de efetividade. Isto porque tais aspectos se relacionam com riscos à saúde geral e auditiva do paciente ${ }^{(22)}$.

Dados do $\mathrm{IBGE}^{(6,18)}$ referem que há aumento de nascimentos provenientes de mães adolescentes. Nessa circunstância, há riscos para a mãe adolescente e para o neonato ${ }^{(13,22,23)}$. Ao se analisar a faixa etária das mães participantes desse estudo, percebe-se que apesar da maior ocorrência ser de mães entre 20 e $29 \operatorname{anos}(47,9 \%, N=572)$, um elevado percentual $(24,6 \%$; 
Tabela 1. Distribuição das mães pesquisadas segundo as variáveis socioeconômicas

\begin{tabular}{|c|c|c|}
\hline Variáveis socioeconômicas & $\mathrm{N}$ & $\%$ \\
\hline \multicolumn{3}{|l|}{ Escolaridade } \\
\hline Analfabeta & 24 & 2,0 \\
\hline $1^{\circ}$ grau incompleto & 431 & 36,1 \\
\hline $1^{\circ}$ grau completo & 333 & 27,9 \\
\hline $2^{\circ}$ grau completo & 301 & 25,3 \\
\hline $3^{\circ} \mathrm{grau}$ & 36 & 3,0 \\
\hline Não informou & 68 & 5,7 \\
\hline \multicolumn{3}{|l|}{ Ocupação } \\
\hline Dona de casa & 664 & 55,7 \\
\hline Estudante & 126 & 10,5 \\
\hline Trabalha fora & 334 & 28,0 \\
\hline Não informou & 69 & 5,8 \\
\hline \multicolumn{3}{|l|}{ Renda pessoal } \\
\hline Não possui renda & 461 & 38,6 \\
\hline <1 salário mínimo & 260 & 21,8 \\
\hline 1 a 3 salários mínimos & 242 & 20,3 \\
\hline >3 salários mínimos & 13 & 1,1 \\
\hline Não informou & 217 & 18,2 \\
\hline \multicolumn{3}{|l|}{ Renda familiar } \\
\hline <1 salário mínimo & 257 & 21,5 \\
\hline 1 a 3 salários mínimos & 7 & 60,6 \\
\hline >3 salários mínimos & 78 & 6,5 \\
\hline Não informou & 135 & 11,3 \\
\hline \multicolumn{3}{|l|}{ Número de filhos } \\
\hline Um & 616 & 51,6 \\
\hline Dois & 284 & 23,8 \\
\hline Três ou mais & 251 & 21,0 \\
\hline Não informou & 42 & 3,5 \\
\hline \multicolumn{3}{|l|}{ Pré-natal } \\
\hline Sim & 1106 & 92,7 \\
\hline Não & 30 & 2,5 \\
\hline Não informou & 57 & 4,8 \\
\hline \multicolumn{3}{|l|}{ Tipo de maternidade } \\
\hline SUS & 1103 & 92,5 \\
\hline Não SUS & 34 & 2,8 \\
\hline Não informou & 56 & 4,7 \\
\hline Total & 1193 & 100,0 \\
\hline
\end{tabular}

Legenda: SUS = sistema único de saúde

$\mathrm{N}=293$ ) das mães eram menores de 20 anos, e apresentava riscos gestacionais ${ }^{(13,22,23)}$. Esses dados estão em concordância com o $\operatorname{IBGE}^{(6,18)}$, que aponta para um aumento de mães adolescentes na região Norte e Nordeste, e servem de alerta para a necessidade de atenção especial aos casos de gravidez na adolescência em nossa região. Complicações na gestação em adolescentes são amplamente divulgadas na literatura e ações de prevenção de doenças, assim como de orientação à saúde, devem ser realizadas principalmente diante de dados reveladores de alta ocorrência gestacional entre adolescentes de uma região ${ }^{(6,18,24)}$

$\mathrm{O}$ apoio financeiro e psicológico de um companheiro tem sido descrito como fundamental para uma boa gestação ${ }^{(23)}$. Neste estudo, foi verificado que 69,2\% (N=825) das mães eram casadas ou viviam em união consensual. Entretanto, 27,8\% $(\mathrm{N}=332)$ das mães relataram ser solteiras. Essa população merece atenção especial, pois estudos alertam para possibilidade crescente de mães solteiras praticarem aborto ${ }^{(8)}$, iniciarem o pré-natal mais tarde ${ }^{(9)}$ e tenderem a iniciar o aleitamento materno mais $\operatorname{tarde}^{(9)}$, aspectos considerados risco para a saúde da gestante e do neonato.

Quanto à escolaridade, verificou-se que 36,1\% (N=431) das mães sequer concluíram o primeiro grau e $2 \%$ são analfabetas. Como o número de anos de instrução formal é considerado um indicador revelador do nível da educação das pessoas ${ }^{(12,13,17)}$, este fator foi considerado como um marcador da condição socioeconômica materna e de sua família, relacionando-se com o perfil cultural e comportamental relacionado aos cuidados de saúde ${ }^{(25)}$. Além disso, o grau de escolaridade das mães é considerado um aspecto que influencia a redução das taxas de fecundidade no país ${ }^{(6)}$.

Um estudo realizado na região nordeste ${ }^{(26)}$ demonstrou que as mães com menor nível de instrução relataram não conhecer a importância do pré-natal e dos fatores de risco para seus bebês. A população com menor escolaridade apresenta menor índice de retorno às consultas, desinformação, menor interesse pelos cuidados com a saúde ou maior dificuldade de acesso aos serviços de atendimento pré-natal.

A escolaridade igual ou menor que quatro anos é, ainda, um fator preditor de baixo peso ao nascer ${ }^{(12)}$. A mãe com este perfil pode não possuir esclarecimentos sobre os cuidados nutricionais necessários durante a gravidez, além do comprometimento da higiene corporal e bucal, assim, como alterações no equilíbrio materno-fetal ${ }^{(13,17,25)}$. Mães com menor grau de escolaridade podem apresentar, mais frequentemente, fatores de risco, para os neonatos, como baixo peso ao nascimento e mortalidade perinatal ${ }^{(13,17,25)}$. Aproximadamente $38 \%$ das mães que participaram do programa de TAN do HC-UFPE possuíam baixa escolaridade, o que sinaliza a necessidade de maior atenção às informações dadas relativas aos cuidados com seu filho.

A baixa escolaridade, associada à pobreza, é um indicador de desinteresse dessas mães em investir nos cuidados com o desenvolvimento do neonato ${ }^{(17,22)}$. Neste aspecto, inclui-se o acompanhamento auditivo, caso seja detectada perda auditiva na criança, através da TAN. Esse grupo torna-se, então, especial no sentido de que sejam criadas estratégias específicas de acompanhamento dos neonatos, minimizando os efeitos devastadores de uma perda auditiva não identificada.

Quanto à ocupação das mães, foi encontrado que 55,7\% $(\mathrm{N}=664)$ relataram ser donas de casa. Esta informação assemelha-se aos resultados referentes à renda pessoal $(43,2 \%$, $\mathrm{N}=495)$. Ou seja, quase metade da população estudada é economicamente ativa. Esse fato faz com que estas mães sejam mais independentes financeiramente da família ou do companheiro $^{(23)}$, pois uma das formas de analisar o seu nível socioeconômico é através de sua renda individual ou familiar ${ }^{(17)}$. 
Por outro lado, observa-se que a outra metade das mães dispõem de uma renda pessoal ou familiar considerada baixa (menor que o salário mínimo), que muitas vezes não satisfaz as necessidades de cuidados da saúde, nutrição e lazer de uma criança ou de uma gestante, prejudicando de maneira global a gestação( ${ }^{(3)}$. Esta análise relaciona-se à saúde de uma população, quando se considera que o acesso a bens e serviços básicos e adequados, no Brasil, ainda depende do nível de rendimento de seus membros. $\mathrm{O}$ conceito de serviços básicos está sendo considerado como os que contribuem para a saúde física, mental e social de uma população e não exclusivamente instituições formais, como hospitais e postos de saúde.

Um aspecto socioeconômico que também merece ser discutido é a realização do pré-natal. O pré-natal é capaz de orientar a promoção da saúde e bem-estar, além de oportunizar o diagnóstico e tratamento de inúmeras complicações que afetam as mães e seus filhos durante a gestação e a redução ou eliminação de fatores e comportamentos de risco passíveis de serem corrigidos ${ }^{(14)}$, como a perda auditiva. A assistência prénatal, no Brasil e em outros países em desenvolvimento, tem se mostrado como um dos principais fatores de proteção contra o baixo peso ao nascer, prematuridade e óbito perinatal ${ }^{(15)}$. Os dados desse estudo revelam uma informação que parece ser positiva e motivadora. Indica que as gestantes acompanhadas e orientadas no HC-UFPE (92\%) estão fora das situações de risco, quando se observa sua saúde e da saúde do feto, o que as protege dos riscos para perda auditiva.

A análise da quantidade de filhos declarada segue a tendência descrita pelo $\operatorname{IBGE}^{(6,18)}$, que mostra que, no Brasil, até 2006, a proporção de mulheres em idade reprodutiva com pelo menos um filho nascido vivo era de $63 \%$. No Estado de Pernambuco, essa proporção foi de $28,3 \%$ e no programa de TAN do HC-UFPE foi de $51,6 \%$.

Dos neonatos e lactentes que participaram do programa de TAN HC-UFPE, mais de $92,5 \%(\mathrm{~N}=1103)$ nasceram em maternidades do SUS. Números semelhantes também foram encontrados em estudo realizado no município de Fortaleza, onde a maior prevalência foi de partos em hospitais públicos ${ }^{(27)}$. Ao se observar que grande parte da população faz uso dos serviços públicos de saúde, é importante que se reflita sobre o investimento governamental em instituições dessa natureza, a fim de que as dificuldades sejam superadas e que sejam oferecidas melhores condições de assistência.

Apesar do SUS ser oferecido a todos os cidadãos, na prática, é utilizado predominantemente por aqueles que possuem menor poder aquisitivo ${ }^{(21)}$. Dessa forma, deve-se buscar um caminho para a melhoria na promoção de saúde populacional e para a identificação de dificuldades, com vistas a planejamentos que possam alcançar resultados de excelência, como todo e qualquer programa de saúde público ou privado.

O diagnóstico dos indicadores populacionais mais frequentes amplia a discussão sobre a situação de carência na assistência de saúde e as reflexões sobre o assunto, sinalizando a necessidade de investigações direcionadas que possam contribuir efetivamente para a solução de dificuldades.

\section{CONCLUSÃO}

A presente pesquisa caracterizou uma população de mães atendidas no Programa de Triagem Auditiva Neonatal, no ano de 2007, do Hospital das Clínicas da Universidade Federal de Pernambuco, quanto a aspectos demográficos e socioeconômicos. Os aspectos estudados são interdependentes, e por isso, muitas vezes, não podem ser considerados de forma isolada.

O conhecimento de tais aspectos socioeconômicos pode levar à realização de ações de atenção básica à saúde que visem minimizar as possíveis causas das alterações auditivas, incentivando melhores condições de saúde. Para o setor público, manter um indivíduo com perda auditiva excluído da sociedade é mais oneroso do que integrá-lo. Esse motivo justifica a implantação, em hospitais públicos e privados, de Programas de Triagem Auditiva Neonatal, atrelados a Programas de Intervenção à Perda Auditiva Neonatal, para que haja identificação de neonatos e lactentes com alteração auditiva o quanto antes, viabilizando sua integração social e ofertando melhor qualidade de vida à sua família.

A análise sobre os resultados dos fatores socioeconômicos revela uma situação considerada desfavorável para a saúde e desenvolvimento global dos neonatos e lactentes, uma vez que as gestantes com o perfil encontrado pertencem a uma categoria com maior probabilidade de risco para perda auditiva e demandam orientações específicas quanto a esses aspectos. Neste aspecto, maior atenção deve ser dada às mães com baixa escolaridade, que não trabalham formalmente e, por conseguinte, não possuem renda pessoal.

Fica clara a necessidade de realização de estudos que avaliem condições demográficas e socioeconômicas relacionando tais aspectos com os resultados encontrados em triagens auditivas realizadas em maternidades. Adicionalmente, reconhece-se a importância de estudos que visem investigar causas e soluções relacionadas às condições socioeconômicas desfavoráveis identificadas na população participante do presente estudo.

\section{ABSTRACT}

\footnotetext{
Purpose: To describe demographic and socioeconomic aspects of mothers of newborns and infants enrolled in the Newborn Hearing Screening Program of the Hospital of the Federal University of Pernambuco. Methods: Data were gathered from the database of the Newborn Hearing Screening Program. Participated in the study 1,193 mothers who delivered babies in 2007. Results: Analysis of the demographic variables showed that most mothers were between 20 and 29 years old (47.9\%), and $69.2 \%$ were married. Socioeconomic variables showed that $36.1 \%$ of the mothers did not complete elementary school, $55.7 \%$ were housewives, $38.6 \%$ did not have any personal income, $60.6 \%$ had familiar income between one and three minimum wages, and $92.5 \%$ gave birth in public maternities. Conclusion: The studied population was considered in unfavorable conditions for the health and the global development
} 
of newborns and infants, for pregnant women within the same socioeconomic status constitute a risk category. These results emphasize the need for the development of health promotion actions for the investigated population.

Keywords: Neonatal screening; Infant; Hearing; Hearing loss; Socioeconomic factors; Population characteristics; Risk index

\section{REFERÊNCIAS}

1. Northern J, Downs MP. Audição na Infância. 5a ed. Rio de Janeiro: Guanabara Koogan; 2005. Avaliação auditiva comportamental; p. 12967.

2. Borges CAB, Moreira LMO, Pena GM, Fernandes FR, Borges BCB, Otani BH. Triagem Auditiva Neonatal Universal. Arq Int Otorrinolaringol(Impr.). 2006;10(1):28-34.

3. Alencar FH, Frota MO. Análise de fatores sócio-econômicosculturais e ambientais relacionados com o défict ponderal de crianças ao nascimento em 1999, em Manaus- AM, Brasil. Acta Amaz. 2003;33(1):33-9.

4. Mukari SZ, Tan KY, Abdullah A. A pilot project on hospital-based universal newborn hearing screening: lessons learned. Int J Pediatr Otorhinolaryngol. 2006;70(5):843-51.

5. American Academy of Pediatrics, Joint Committee on Infant Hearing. Year 2007 position statement: Principles and guidelines for early hearing detection and Intervention programs. Pediatrics. 2007;120(4):898-921.

6. Brasil. Fundação Instituto de Geografia e Estatística (IBGE). Uma Análise das Condições de Vida da População Brasileira. Brasileira. Estudos e Pesquisas Informações Demográficas e Sócio-Econômica. Rio de Janeiro: IBGE. Síntese de Indicadores Sociais, 21; 2007. [Internet]. [citado 2008 Mar 28]; Disponível em: http://www1.ibge. gov.br/home/estatistica/populacao/condicaodevida/indicadoresminimos/ sinteseindicsociais2007/indic_sociais2007.pdf

7. Brandão ER, Heilborn ML. Sexualidade e gravidez na adolescência entre jovens de camadas médias do Rio de Janeiro, Brasil. Cad. Saúde Pública. 2006;22(7):1421-30.

8. Kac G, Silveira EA, Oliveira LC, Araujo DMR, Sousa EB. Fatores associados à ocorrência de cesárea e aborto em mulheres selecionadas em um centro de saúde no município do Rio de Janeiro, Brasil. Rev Bras Saúde Matern Infant. 2007;7(3):271-80.

9. Venâncio SI, Escuder MML, Kitoko P, Rea MF, Monteiro CA. Frequência e determinantes do aleitamento materno em municípios do Estado de São Paulo. Rev Saúde Pública. 2002;36(3):313-8.

10. Sabroza AR, Leal MC, Gama SGN, Costa JV. Perfil sócio-demográfico e psicossocial de puérperas adolescentes do Município do Rio de Janeiro, Brasil - 1999-2001. Cad Saúde Pública. 2004;20(Suppl.1):S112-S120.

11. Todd NW. Universal newborn hearing screening follow-up in two Georgia populations: newborn, mother and system correlates. Int J Pediatr Otorhinolaryngol. 2006;70(5):807-15.

12. Cruz SS, Costa MCN, Gomes Filho IS, Vianna MIP, Santos CT. Doença periodontal materna como fator associado ao baixo peso ao nascer. Rev Saúde Publica. 2005;39(5):782-7.

13. Aquino TA, Guimaraes MJB, Sarinho SW, Ferreira LOC. Fatores de risco para a mortalidade perinatal no Recife, Pernambuco, Brasil, 2003. Cad Saúde Pública. 2007;23(12):2853-61.

14. Kilsztajn S, Rossbach A, Carmo MSN, Sugahara GTL. Assistência prénatal, baixo peso e prematuridade no Estado de São Paulo, 2000. Rev Saúde Pública. 2003;37(3):303-10.

15. Gama SGN, Szwarcwald CL, Leal MC. Experiência de gravidez na adolescência, fatores associados e resultados perinatais entre puérperas de baixa renda. Cad Saúde Pública. 2002;18(1):153-61.
16. Fonseca TMV, Cesar JA, Hackenhaar AA, Ulmi EF, Neumann NA. Corrimento vaginal referido entre gestantes em localidade urbana no Sul do Brasil: prevalência e fatores associados. Cad Saúde Pública. 2008;24(3):558-66.

17. Andrade CLT, Szwarcwald CL, Gama SGN, Leal MC. Desigualdades sócio-econômicas do baixo peso ao nascer e da mortalidade perinatal no Município do Rio de Janeiro, 2001. Cad Saúde Pública. 2004;20(Suppl. 1):S44-S51.

18. Brasil. Fundação Instituto de Geografia e Estátistica (IBGE). Educação melhora, mas ainda apresenta preocupações. Rio de Janeiro: IBGE. Síntese de Indicadores Sociais, 22; 2008. [Internet]. [citado 2008 Set 24]; Disponível em: http://www.ibge.gov.br/home/presidencia/noticias/ noticia_visualiza.php?id_noticia $=1233 \& i d \_p a g i n a=1$.

19. Leal MC, Gama SGN, Ratto KMN, Cunha CB. Uso do índice de Kotelchuck modificado na avaliação da assistência pré-natal e sua relação com as características maternas e o peso do recém-nascido no Município do Rio de Janeiro. Cad Saúde Pública. 2004;20(Suppl. 1):S63-S72.

20. Calvasina PG, Nations MK, Jorge MSB, Sampaio HAC. "Fraqueza de nascença": sentidos e significados culturais de impressões maternas na saúde infantil no Nordeste brasileiro. Cad Saúde Pública. 2007;23(2):371-80.

21. Baraldi ACP, Daud ZP, Almeida AM, Gomes FA, Nakano AMS. Gravidez na adolescência: estudo comparativo das usuárias das maternidades públicas e privadas. Rev Latinoam Enferm. 2007;15(n. especial):799-805.

22. Lima MLLT, Assis ABR, Mercês GB, Barros PF, Griz SMS. Triagem auditiva: perfil socioeconômico de mãe. Rev CEFAC. 2008;10(2):25460.

23. Carniel EF, Zanolli ML, Almeida CAA, Morcillo AM. Características das mães adolescentes e de seus recém-nascidos e fatores de risco para a gravidez na adolescência em Campinas, SP, Brasil. Rev Bras Saúde Matern Infant. 2006;6(4):419-26.

24. Cabral CS. Contracepção e gravidez na adolescência na perspectiva de jovens pais de uma comunidade favelada do Rio de Janeiro. Cad Saúde Pública. 2003;19(suppl. 2):S283-S292.

25. Silva CF, Leite AJM, Almeida NMGS, Gondim RC. Fatores de risco para mortalidade infantil em município do Nordeste do Brasil: linkage entre bancos de dados de nascidos vivos e óbitos infantis: 2000 a 2002. Rev Bras Epidemiol. 2006;9(1):69-80.

26. Lima GSP, Sampaio HAC. Influência de fatores obstétricos, socioeconômicos e nutricionais da gestante sobre o peso do recémnascido: estudo realizado em uma maternidade em Teresina, Piauí. Rev Bras Saúde Matern Infant. 2004;4(3):253-61.

27. Castro, ECM. Morbimortalidade hospitalar de recém-nascidos de muito baixo peso no município de Fortaleza [dissertação]. [Fortaleza(CE)]: Universidade Federal do Ceará; 2004. 